\title{
Variabilidad genética de familias de medios hermanos de melón criollo ecuatoriano Cucumis melo var. dudaim (L.) Naudin
}

\section{Genetic variability of families of half-sibling of ecuadorian creole melon Cucumis melo var. dudaim (L.) Naudin}

\author{
José Francisco Espinosa-Carillo ${ }^{1}$; Franco Alirio Vallejo-Cabrera ${ }^{2}$
}

1'Ing. Agrónomo. Universidad Técnica Estatal de Quevedo, Campus "Ingeniero Manuel Agustín Haz Álvarez", Unidad de Estudios a Distancia, Carrera de Ingeniería Agropecuaria. Quevedo - Provincia de Los Ríos, Ecuador; e-mail: jespinosa@uteq.edu.ec; (D) https://orcid.org/0000-0003-0161-6483

${ }^{2}$ Ing. Agrónomo. Ph.D. Universidad Nacional de Colombia, Sede Palmira, Facultad de Ciencias Agropecuarias. Palmira - Valle del Cauca, Colombia; e-mail: favallejoc@unal.edu.co; (D) https://orcid.org/0000-0002-2739-0745

*autor para correspondencia: jespinosa@uteq.edu.ec

Cómo citar: Espinosa-Carillo, J.F.; Vallejo-Cabrera, F.A. 2020. Variabilidad genética de familias de medios hermanos de melón criollo ecuatoriano Cucumis melo var. dudaim (L.) Naudin. Rev. U.D.C.A Act. \& Div. Cient. 23(2):e1762. http://doi.org/10.31910/rudca.v23. n2.2020.1762

Artículo de acceso abierto publicado por Revista U.D.C.A Actualidad \& Divulgación Científica, bajo una licencia Creative Commons CC BY-NC 4.0

Publicación oficial de la Universidad de Ciencias Aplicadas y Ambientales U.D.C.A, Institución de Educación Superior Acreditada de Alta Calidad por el Ministerio de Educación Nacional.

Recibido: Abril 12 de 2019 Aceptado: Diciembre 1 de 2020 Editado por: Ingeborg Zenner de Polanía

\section{RESUMEN}

El melón, se cultiva en climas templados y cálidos de todo el mundo; no obstante, la información sobre la variabilidad genética del melón criollo ecuatoriano es limitada, para iniciar programas de mejoramiento. El objetivo de esta investigación fue evaluar características de producción y de calidad del fruto, en 20 familias de medios hermanos, seleccionadas de melón criollo ecuatoriano. El estudio, se realizó en el cantón Palenque, Ecuador. La semilla de las 20 familias seleccionadas, se dispusieron en bloques completos al azar, con tres repeticiones. Las diferencias entre familias fueron significativas para siete caracteres. La familia 73 presentó mayor peso, diámetro, longitud, espesor, cavidad del fruto y producción por hectárea; la familia 7 obtuvo el mayor promedio para grosor de corteza, sólidos solubles y firmeza de pulpa, mientras en la familia 196, se destacó la acidez de pulpa. Existen asociaciones fenotípicas altamente significativas entre peso de fruto con diámetro, longitud, espesor de pulpa, cavidad de fruto y producción por hectárea, en donde los coeficientes de variación genotípica fueron mayores que los coeficientes de variación ambiental. Las estimaciones de heredabilidad en sentido amplio fueron de magnitud alta $(>60 \%)$, para los 10 caracteres evaluados y la magnitud del progreso genético esperado fue alta $(>20 \%)$, para peso del fruto, producción por hectárea y grosor de corteza; moderada, para cavidad del fruto y sólidos totales. La selección para melones variedad dudaim, se debe centrar en contenido de sólidos solubles y peso del fruto, que presentó una alta correlación con el rendimiento.

Palabras clave: Variación genética; Variación ambiental; Heredabilidad; Progreso genético.

\section{ABSTRACT}

Melon is an important horticultural crop in temperate and warm climates around the world; however, information on the genetic variability of ecuadorian creole melon is limited to initiate breeding 
programs. The objective of this research was to evaluate 20 families of half-sibling of ecuadorian creole melon by characteristics of production and fruit quality. The study was carried out in Palenque canton, Ecuador. The seed of the 20 families selected was arranged in complete blocks at random with three repetitions. The differences between families were significant for seven characters. Family 73 had greater weight, diameter, length, thickness, fruit cavity, and production per hectare, family 7 had the highest average for bark thickness, soluble solids and pulp firmness; the 196 family for pulp acidity. There are highly significant phenotypic associations between fruit weight with diameter, length, pulp thickness, fruit cavity and production per hectare. The coefficients of genotypic variation were greater than the coefficients of environmental variation. The estimates of heritability in the broad sense were of high magnitude $(>60 \%)$ for the 10 characters evaluated and the magnitude of the expected genetic progress was high $(>20 \%)$ for fruit weight, production per hectare, and bark thickness; moderate for fruit cavity and total solids; the selection for melons of the dudaim variety should focus on soluble solids content and fruit weight that showed a high correlation with yield.

Keywords: Genetic variation; Environmental variation; Heritability; Genetic progress.

\section{INTRODUCCIÓN}

El melón (Cucumis melo L.) es un importante cultivo hortícola en todo el mundo, tanto en climas templados como cálidos. El género Cucumis pertenece a la familia de las Cucurbitáceas y está representada por 32 especies; C. melo es una especie morfológicamente diversa, compuesta por poblaciones silvestres y domesticadas (Soltani et al. 2010).

El género Cucumis, tiene numerosas especies silvestres en el continente africano, posiblemente, su centro de origen (Sebastian et al. 2010)which includes cucumber (Cucumis sativus, de donde se dispersó al resto del mundo. Actualmente, es cultivado bajo condiciones climáticas tropicales y subtropicales (Reddy et al. 2013).

En las provincias del Manabí, Los Ríos y Guayas, se han identificado melones criollos, uno de ellos es el conocido como melón de Bolsillo o de Mano, que pertenece a la variedad dudaim y se lo conoce con los nombres comunes de Pomegranate melon, Chito melon, Queen Anne's pocket melon y melón Mango; en la clasificación de Guis corresponde a C. melo var. dudaim Naud (Guis et al. 1998) (Figura 1). Estos melones presentan frutos de forma redonda, tamaño pequeño, superficie lisa, sabor aromático fuerte y poco dulce (Pitrat et al. 2000) (Figura 2). Son valorados por su olor exótico y belleza ornamental (Paris et al. 2012).

En la costa ecuatoriana, el germoplasma del melón criollo ecuatoriano es cultivado y conservado por pequeños agricultores, además es muy apreciado por los consumidores locales. A pesar de ser una variedad poco explorada exhibe una amplia diversidad fenotípica para los rasgos del fruto, como maduración, forma, tamaño, color de la pulpa, textura, dulzura y aroma. Por referencias de los productores de esta fruta, se tiene que la producción promedio de esta variedad criolla está por debajo de las 7 t.ha ${ }^{-1}$, muy inferior a la producción de los híbridos importados, como el Honey Dew, que se cultiva comercialmente (INEC, 2017); datos de FAO (2018), basados en metodología de imputación, indicaron que, en Ecuador, en el 2016, se cultivaron 2.036 hectáreas, con un rendimiento promedio de $2,43 \mathrm{~kg} / \mathrm{m}^{2} ;$ sin embargo, no se han realizado investigaciones que permitan mejorar, tanto la calidad de los frutos como la productividad de este cultivo.

Para mantener y mejorar la competitividad del melón variedad dudaim es necesario promover el mejoramiento de estos cultivares, para que ofrezcan mejores características productivas y organolépticas, además, se debe generar nuevas tecnologías de manejo del cultivo.

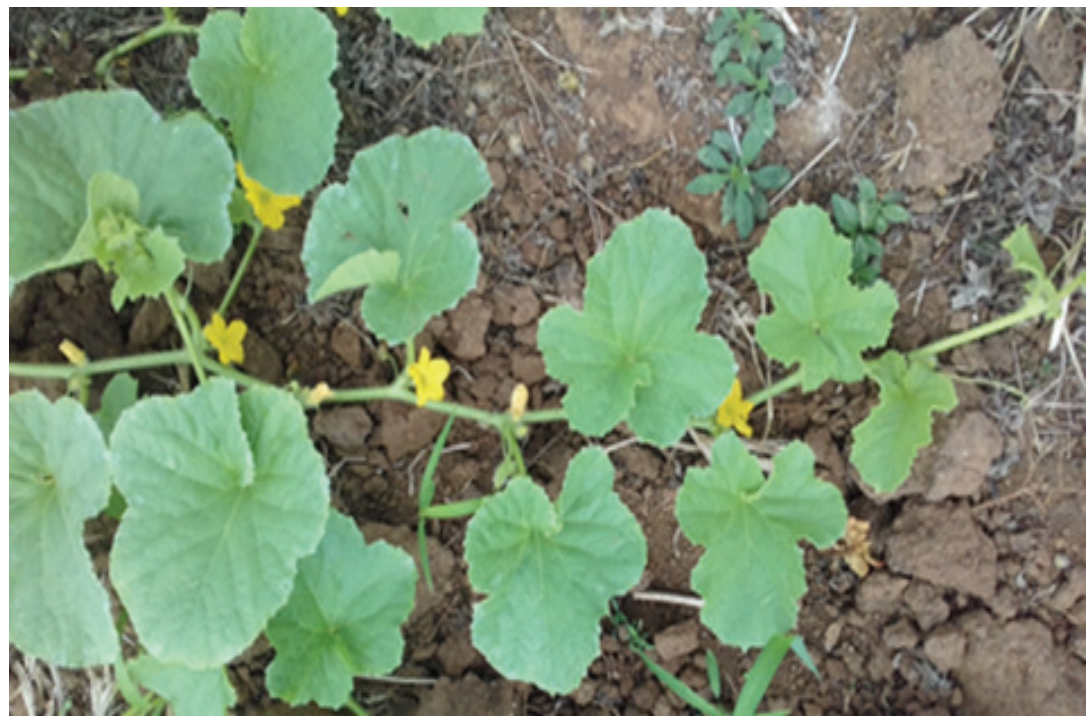

Figura 1. Planta del melón criollo ecuatoriano Cucumis melo var. dudaim (L.) Naudin. 

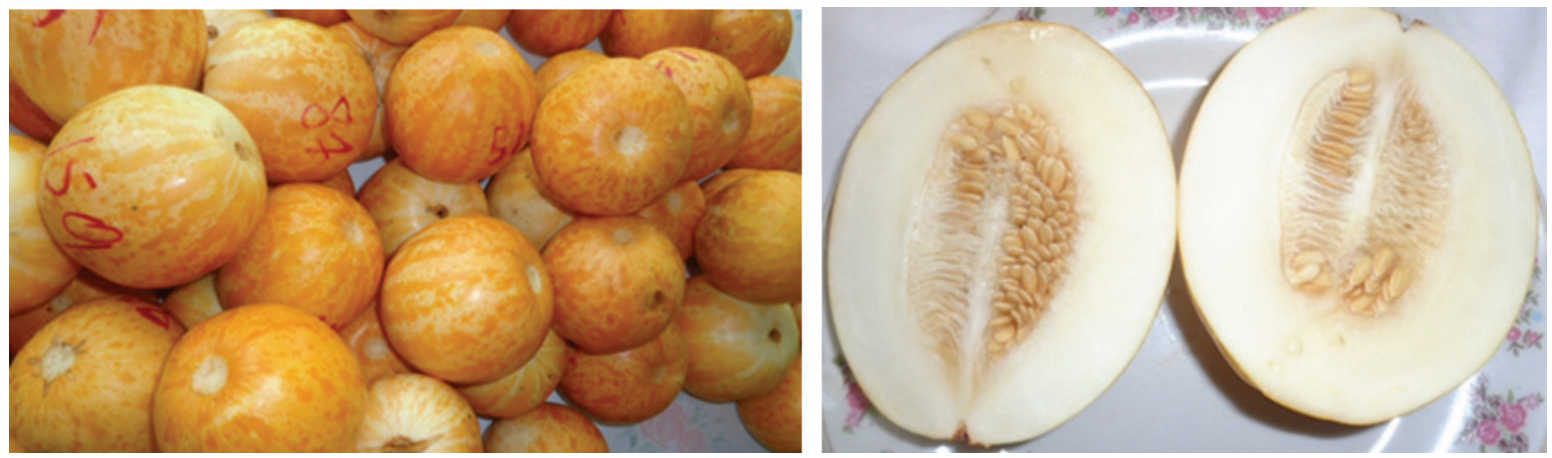

Figura 2. Fruto del melón criollo ecuatoriano Cucumis melo var. dudaim (L.) Naudin.

El mejoramiento genético está orientado a obtener cultivares con frutos adaptados a los gustos del consumidor y a las exigencias de la comercialización (Vallejo \& Estrada, 2004). La selección de familias de medios hermanos, se ha introducido para hacer más eficiente el incremento de la frecuencia de alelos favorables para caracteres de baja heredabilidad (Peña et al. 2013).

El potencial productivo del melón criollo de Mano, para alcanzar avances en la producción y calidad organoléptica, debe ser probado y estudiado mediante procedimientos formales. En la presente investigación, se evaluaron 20 familias seleccionadas de medios hermanos de melón criollo ecuatoriano Cucumis melo var. dudaim (L.) Naudin, con el objetivo de medir la variabilidad genética de las características deseables del fruto, principalmente, de producción y de calidad.

\section{MATERIALES Y MÉTODOS}

Los trabajos de campo, se realizaron en el recinto Embarcadero del cantón Palenque, provincia de Los Ríos, ubicado a una altitud de $31 \mathrm{~m}$ s.n.m., en las coordenadas de latitud $1^{\circ} 26^{\prime} 12^{\prime \prime} \mathrm{S}$ y longitud $79^{\circ} 45^{\prime} 21^{\prime \prime} \mathrm{O}$, caracterizado, climáticamente, por una temperatura promedio de $24-25^{\circ} \mathrm{C}$, humedad relativa promedio de $80 \%, 1011,9$ horas luz anuales, precipitaciones de $1500-2000 \mathrm{~mm}$ al año y pertenece a la zona ecológica de Bosque Seco Tropical (Gobierno Autónomo Descentralizado del Cantón Quevedo, 2014; Instituto Nacional de Meteorologia e Hidrologia INAMHI, 2018).

Se utilizó semilla de 20 familias de medios hermanos de melón criollo ecuatoriano, proveniente de frutos de plantas seleccionadas en un estudio previo. Como tratamiento control, se utilizó semilla de la población original (Po) colectada.

Las 20 familias medios hermanos seleccionadas y el tratamiento control (Po), se evaluaron en un diseño de bloques completos al azar con tres repeticiones. En cada repetición, las familias se ubicaron aleatoriamente. Las unidades experimentales fueron parcelas lineales con 5 plantas, la parcela efectiva quedó constituida por 3 plantas, a una separación de $3 \mathrm{~m}$ entre surcos y 1,50m entre plantas, de acuerdo con la forma tradicional de cultivo de melones criollos; la densidad de población fue de 2.222 plantas por hectárea.
En cada planta, se evaluaron los caracteres relacionados con el fruto comercial: peso de fruto $(\mathrm{PF})$, usando una balanza electrónica, marca Jadever JWP, de 6000,0 \pm 0,1g de capacidad, diámetro de fruto (DF), longitud de fruto (LF), espesor de la pulpa (EP) (Wang et al. 2016) grosor de la corteza (GC) y cavidad del fruto (CF) (Aragão et al. 2015), medidas con un calibrador digital electrónico marca Uberman; firmeza de la pulpa (FP), cuantificada con un medidor de dureza de fruto modelo FHT-804, con capacidad de $24 \pm 0,1 \mathrm{~kg}$ $\mathrm{cm}^{2}$ (Monge, 2016); (SS) sólidos solubles ( ${ }^{\circ}$ Brix), medidos con un refractómetro RHB-50ATC, escala de 0,0-50,0 $\pm 0,2 \%$ (Aragão et al. 2015); acidez de la pulpa (AP), cuantificada con un medidor electrónico digital de $\mathrm{pH}$, marca Biocharge ATC y, producción por hectárea, calculado con el peso medio de los frutos en densidad poblacional de 2.222 plantas por hectárea.

Los resultados experimentales obtenidos de cada carácter evaluado fueron sometidos a un análisis de varianza con el software estadístico SAS versión 9.4 - 2019 (Statistical Analysis System); se realizó la comparación de promedios, mediante la prueba de Tukey ( $\mathrm{p} \leq$ 0,05) (Abraham-Juárez et al. 2018), además, se efectuó análisis de correlación entre los caracteres evaluados.

Se determinaron las varianzas ambientales, genéticas y fenotípicas utilizando el análisis de varianza, según las ecuaciones abajo indicadas: Varianza ambiental: $\left(\sigma_{\mathrm{A}}^{2}\right)=\mathrm{CMe} / \mathrm{r}$

Varianza genética: $\left(\sigma_{\mathrm{G}}^{2}\right)=\mathrm{CMf}-\mathrm{CMe} / \mathrm{r}$

Varianza fenotípica: $\left(\sigma_{\mathrm{F}}^{2}\right)=\sigma_{\mathrm{G}}^{2}+\sigma^{2}{ }_{\mathrm{A}}$

Donde: $\mathrm{CMf}=$ cuadrado medio de las familias, $\mathrm{CMe}=$ cuadrado medio del error experimental y $\mathrm{r}=$ número de repeticiones (Pistorale et al. 2008).

Los coeficientes de variación genética y ambiental, se calcularon según:

Coeficiente de variación genética: $\mathrm{CVg}=\frac{\sqrt{\sigma^{2} \mathrm{G}}}{\overline{\mathrm{x}}} \times 100$

Coeficiente de variación ambiental: $\mathrm{CVa}=\frac{\sqrt{\sigma^{2} \mathrm{~A}}}{\overline{\mathrm{x}}} \times 100$ 
Heredabilidad en sentido amplio $\left(\mathrm{h}^{2} \mathrm{~A}\right)$ para cada variable fue estimada de la forma clásica, como se describe a continuación: $\mathrm{h}_{\mathrm{A}}^{2}=\left(\sigma_{\mathrm{G}}^{2} / \sigma_{\mathrm{F}}^{2}\right) 100$

Progreso genético esperado, con la fórmula clásica: $h^{2}$, donde ds $=$ diferencial de selección.

Los valores de los coeficientes de variación fenotípica (CVf), variación genotípica $(\mathrm{CVg})$ y avance genético, se clasificaron como bajos $(<10 \%)$, moderados $(10-20 \%)$ y altos $(>20 \%)$. Los valores de heredabilidad, se clasificaron como bajos $(<30 \%)$, moderados (30-60\%) y altos (>60\%), valores utilizados por Reddy et al. (2013).

El establecimiento de las parcelas, se realizó mediante siembra directa, depositando una semilla por sitio, previamente tratada con Fosetil aluminio (i.a. Fosetil Aluminio Aluminium tris-oethylphosphonato). El cultivo recibió la fertilización de $150 \mathrm{~kg} \mathrm{ha}^{-1}$ de la fórmula 8-20-20 más $100 \mathrm{~kg} \mathrm{ha}^{-1}$ de urea $\mathrm{N}$ al $46 \%$, en dos aplicaciones. Se realizó el control de malezas en premergencia, con una aplicación de Glifosato (i.a. glifosato) y en pos emergencia, una aplicación de Gramoxone ${ }^{\circledR}$ Super (i.a. Dicloruro de Paraquat $276 \mathrm{~g}$ $\mathrm{L}^{-1}$ ), entre plantas, más una aplicación de Verdict (i.a. HaloxyfopR-metil éster108 $\mathrm{L}^{-1}$ ); adicionalmente, se realizaron dos limpiezas manuales. El control de plagas, principalmente trips (Frankliniella occidentalis) y pulgón (Aphididae), se realizó con seis aplicaciones rotativas de Crisabamet (i.a. abamectin), Confidor (i.a. imidacloprid) y Alistin (i.a. triflumuron). El control de enfermedades fungosas, se realizó en forma preventiva en las ocho primeras semanas del cultivo, con ocho aplicaciones rotativas de Fosetil aluminio y Maxim (i.a. fludioxonil 2,5g + metalaxil-M 1,0g). El suelo, se mantuvo en capacidad de campo, mediante un sistema de riego por goteo, instalado con goteros, que suministraron agua en caudal de $2 \mathrm{~L}$ por hora. La cosecha de los frutos, se inició a los 93 días después de la siembra.

\section{RESULTADOS Y DISCUSIÓN}

Los valores del cuadrado medio de los 10 caracteres evaluados en las 20 familias de medios hermanos de melón variedad dudaim, presentaron diferencias altamente significativas $\mathrm{P} \leq 0,01$, entre familias, para peso del fruto (PF), diámetro del fruto (DF), longitud del fruto (LF), cavidad del fruto (CF), acidez de la pulpa (AP), (SS) sólidos solubles ( ${ }^{\circ}$ Brix) y producción por hectárea $\left(\mathrm{kg} \mathrm{ha}^{-1}\right)$. No se presentaron diferencias estadísticas en las variables grosor de la corteza (GC), espesor de la pulpa (EP), firmeza de la pulpa (FP). El coeficiente de variación (CV) fue superior al 39\% para peso del fruto $(\mathrm{PF})$, firmeza de la pulpa (FP) y producción por hectárea $\left(\mathrm{kg} \mathrm{ha}^{-1}\right)$; estos coeficientes de variación ponen de manifiesto la diversidad de respuestas para estas variables; los demás caracteres evaluados presentaron un (CV) inferior al 15\% (Tabla 1).

Tabla 1. Cuadrados medios del análisis de varianza y coeficientes de variación, para 10 caracteres relacionados con el fruto de melón criollo ecuatoriano Cucumis melo var. dudaim (L.) Naudin

\begin{tabular}{|l|c|c|c|c|c|c|}
\hline F de V. & $\mathbf{G L}$ & $\mathbf{( P F}) \mathbf{g}$ & $\mathbf{( D F}) \mathbf{m m}$ & $\mathbf{( L F}) \mathbf{m m}$ & $\mathbf{( E P}) \mathbf{m m}$ & $(\mathbf{G C}) \mathbf{m m}$ \\
\hline Bloques & 2 & $59498,44^{* *}$ & $14,31^{* *}$ & $126,08^{\text {ns }}$ & $1,13^{\text {ns }}$ & $0,01^{\text {ns }}$ \\
\hline Familias & 20 & $29508,85^{* *}$ & $105,76^{* *}$ & $268,28^{* *}$ & $6,45^{\text {ns }}$ & $0,01^{\text {ns }}$ \\
\hline Bloque x Familia & 40 & 8249,90 & 32,02 & 92,38 & 4,12 & 0,01 \\
\hline CV & & 29,39 & 7,45 & 11,70 & 11,57 & 13,45 \\
\hline
\end{tabular}

\begin{tabular}{|c|c|c|c|c|c|c|}
\hline F de V. & GL & (CF) $\mathrm{mm}$ & (FP) $\mathrm{kg} \mathrm{cm}^{2}$ & $(\mathrm{AP}) \mathrm{pH}$ & (SS) $\left({ }^{\circ}\right.$ Brix $)$ & (P) $\left(\mathrm{kg} \mathrm{ha}^{-1}\right)$ \\
\hline Bloques & 2 & $8,29 \mathrm{~ns}$ & $7,83^{\text {ns }}$ & $0,01^{\mathrm{ns}}$ & $0,10^{\mathrm{ns}}$ & $3197161,22^{*}$ \\
\hline Familias & 20 & $92,98 * *$ & $6,71^{\mathrm{ns}}$ & $0,02 * *$ & $32,43^{* *}$ & $1169703,73^{*}$ \\
\hline Bloque x Familia & 40 & 12,80 & 5,56 & 0,01 & 0,81 & 661421,14 \\
\hline $\mathrm{CV}$ & & 8,46 & 29,89 & 1,42 & 8,87 & 46,32 \\
\hline
\end{tabular}

F de V: Fuente de variación, GL: grados de libertad, **: significancia nivel $\mathrm{P}<0,01$, *: significancia nivel $\mathrm{P}<0,05$ de probabilidad, ns: no significativo, peso del fruto (PF), diámetro del fruto (DF), longitud del fruto (LF), espesor de la pulpa (EP), grosor de la corteza (GC), cavidad del fruto (CF), firmeza de la pulpa (FP), acidez de la pulpa (AP), (SS) sólidos solubles ( ${ }^{\circ}$ Brix) y (P) producción por hectárea (kg ha ${ }^{-1}$ ). 
Estos resultados indicaron que las poblaciones criollas contienen amplias reservas de variación genética y adaptación a diferentes condiciones del ambiente (Eguiarte et al. 2015). También indicaron la existencia de posibilidades de mejoramiento, por el aprovechamiento de los efectos genéticos aditivos y no aditivos, a través de la selección de plantas con atributos de fruto favorables (Aramendiz et al. 2014) y concuerdan con los resultados obtenidos por Reddy et al. (2013), cuando afirmaron que las diferencias altamente significativas entre los genotipos indican presencia de suficiente cantidad de variabilidad en el germoplasma, facilitando las posibilidades de selección, para modificar los caracteres en estudio.

Los promedios, valores máximos, mínimos y diferencia mínima significativa para los caracteres de las 20 familias de C. melo var. dudaim, se presentan en la tabla 2.

Tabla 2. Promedios, valores máximos, mínimos y diferencia mínima significativa, para 10 caracteres de 20 familias de medios hermanos de melón criollo ecuatoriano Cucumis melo var. dudaim (L.) Naudin

\begin{tabular}{|c|c|c|c|c|c|c|c|c|c|c|}
\hline \multirow[b]{2}{*}{ Familias } & \multicolumn{10}{|c|}{ Características relacionadas con los frutos } \\
\hline & $\begin{array}{c}(\mathrm{PF}) \\
\mathrm{g}\end{array}$ & $\begin{array}{l}\text { (DF) } \\
\mathrm{mm}\end{array}$ & $\begin{array}{l}\text { (LF) } \\
\mathrm{mm}\end{array}$ & $\begin{array}{l}\text { (EP) } \\
\mathrm{mm}\end{array}$ & $\begin{array}{l}\text { (GC) } \\
\mathrm{mm}\end{array}$ & $\begin{array}{l}\text { (CF) } \\
\mathrm{mm}\end{array}$ & $\begin{array}{c}(\mathrm{SS}) \\
\left({ }^{\circ} \mathrm{Brix}\right)\end{array}$ & $\begin{array}{c}\text { (AP) } \\
\mathrm{pH}\end{array}$ & $\underset{\mathrm{kg} \mathrm{cm}^{-2}}{(\mathrm{FP})}$ & $\begin{array}{c}(\mathrm{P}) \\
\left(\mathrm{kg} \mathrm{ha}^{-1}\right)\end{array}$ \\
\hline 5 & 475,39 & 80,38 & 85,66 & 0,70 & 18,13 & 43,82 & 10,39 & 6,34 & 8,21 & 2112,61 \\
\hline 7 & 333,00 & 77,26 & 87,97 & 0,77 & 18,28 & 39,48 & 11,83 & 6,32 & 9,25 & 1806,36 \\
\hline 10 & 261,79 & 78,27 & 82,40 & 0,62 & 18,17 & 40,54 & 9,78 & 6,46 & 3,59 & 1405,96 \\
\hline 15 & 400,21 & 80,47 & 95,52 & 0,64 & 18,78 & 42,20 & 9,11 & 6,28 & 4,99 & 3063,78 \\
\hline 34 & 484,08 & 80,76 & 88,85 & 0,73 & 18,57 & 47,44 & 8,61 & 6,34 & 4,52 & 2752,47 \\
\hline 45 & 202,31 & 73,18 & 78,59 & 0,68 & 16,32 & 40,18 & 11,11 & 6,31 & 6,77 & 1206,86 \\
\hline 49 & 331,87 & 73,94 & 77,75 & 0,61 & 16,47 & 38,82 & 9,94 & 6,49 & 7,36 & 1918,70 \\
\hline 51 & 267,90 & 72,75 & 78,87 & 0,67 & 16,88 & 40,85 & 9,89 & 6,28 & 8,01 & 1830,52 \\
\hline 68 & 253,98 & 72,57 & 76,79 & 0,67 & 17,13 & 37,80 & 10,89 & 6,51 & 7,92 & 1805,04 \\
\hline 72 & 264,44 & 74,87 & 80,57 & 0,68 & 16,79 & 40,54 & 10,58 & 6,25 & 5,80 & 389,74 \\
\hline 73 & 566,90 & 97,38 & 115,31 & 0,69 & 22,60 & 62,45 & 8,50 & 6,26 & 5,35 & 3076,77 \\
\hline 76 & 201,99 & 72,73 & 77,44 & & 16,08 & 39,16 & 10,17 & & 53 & 990,86 \\
\hline 78 & 250,53 & 75,60 & 79,46 & & 17,13 & 40,43 & 10,67 & & 6,45 & 1113,37 \\
\hline 79 & 202,36 & 70,13 & 71,70 & 0,64 & 16,36 & 36,94 & 11,17 & 6,43 & 6,76 & 1438,77 \\
\hline 81 & 351,48 & 71,07 & 77,40 & 0,7 & 16,00 & 37,82 & 10,33 & 6,41 & 7,66 & 1561,99 \\
\hline 83 & 277,24 & 77,13 & 84,21 & 0,61 & 18,26 & 40,08 & 9,78 & 6,42 & 7,92 & 1317,09 \\
\hline 116 & 346,04 & 72,79 & 76,31 & 0,57 & 16,96 & 39,47 & 10,56 & 6,40 & 46 & 2478,35 \\
\hline 147 & 272,45 & 77,63 & 84,14 & 0,59 & 17,92 & 39,46 & 10,61 & 6,29 & 5,36 & 1324,43 \\
\hline 175 & 267,77 & 75,82 & 77,90 & 0,62 & 18,22 & 38,68 & 10,56 & 6,41 & 5,56 & 1774,69 \\
\hline 194 & 243,36 & 70,97 & 75,14 & 0,55 & 16,07 & 39,14 & 10,33 & 6,43 & 4,68 & 1463,85 \\
\hline Po & 234,35 & 70,17 & 73,64 & 0,68 & 17,16 & 34,49 & 8,39 & 6,44 & 6,29 & 1041,44 \\
\hline Media & 309,02 & 75,99 & 82,17 & 0,64 & 17,54 & 40,94 & 10,15 & 6,37 & 6,54 & 1755,89 \\
\hline Máximo & 566,90 & 97,38 & 115,31 & 0,77 & 22,60 & 62,45 & 11,83 & 6,51 & 9,25 & 3076,77 \\
\hline Mínimo & 201,99 & 70,13 & 71,70 & 0,52 & 16,00 & 34,49 & 8,39 & 6,25 & 3,59 & 990,86 \\
\hline DMS & 67,181 & 4,3164 & 7,2276 & 1,5024 & 0,0685 & 2,6876 & 1,7706 & 0,0649 & 0,6052 & 603,92 \\
\hline
\end{tabular}

Peso del fruto (PF), diámetro del fruto (DF), longitud del fruto (LF), espesor de la pulpa (EP), grosor de la corteza (GC), cavidad del fruto $(\mathrm{CF})$, firmeza de la pulpa (FP), acidez de la pulpa (AP) (SS) sólidos solubles $\left({ }^{\circ} \mathrm{Brix}\right)$ y $(\mathrm{P})$ producción por hectárea $(\mathrm{kg} / \mathrm{ha}), \mathrm{DMS}=$ diferencia mínima significativa Tukey $(\mathrm{P}<0,05)$. 
La familia 73 presentó mayor peso promedio de fruto, diámetro de fruto, longitud del fruto, espesor de la pulpa, cavidad del fruto y producción por hectárea, con valores que son superiores a los del tratamiento control, que corresponde a la población original (Po) antes de selección.

La familia 7 presentó mayor promedio para grosor de la corteza, sólidos solubles y firmeza de la pulpa. El valor de grados Brix es superior a lo reportado por Pérez-Zamora et al. (2007), quienes en su estudio, realizado con melón cantaloupoe, encontraron valores entre 9,54 a 9,74 y, ligeramente menor, a lo reportado por CanoRíos et al. (2004), en su estudio de calidad de la fruta de híbridos de melón, donde el híbrido cruiser, presentó $11,00^{\circ}$. En firmeza de la pulpa, el valor encontrado supera a lo que consideran los autores: según Díaz-Alvarado \& Monge-Pérez (2017), en los frutos de melón, considera como valor óptimo 2,0 o más kg/ $\mathrm{cm}^{2}$ (Monge, 2016), la firmeza mínima aceptable es de $3,1 \mathrm{~kg} / \mathrm{cm}^{2}$, para melón Cantaloupe y Orange Flesh. De igual manera, la familia 68 presentó el mayor promedio de acidez de la pulpa $(6,51 \mathrm{pH})$, valor que es ligeramente superior a lo reportado por Retamal et al. (2013), donde los melones Cantaolupe, en condiciones normales, presentaron un $\mathrm{pH}$ alrededor de 6,3.

Los caracteres que mostraron una amplia variabilidad entre las familias de medios hermanos son el peso promedio del fruto (566,90 - 201,99g), diámetro del fruto (97,38 - 70,13mm), longitud del fruto $(115,31-71,70 \mathrm{~mm})$, cavidad del fruto $(62,45-34,49 \mathrm{~mm})$, espesor de la pulpa $(22,60-16,00 \mathrm{~mm})$, producción por superficie $\left(3076,77\right.$ - 990,86kg ha $\left.{ }^{-1}\right)$ y firmeza de la pulpa $\left(9,25-3,59 \mathrm{~kg} \mathrm{~cm}^{2}\right)$. La firmeza de pulpa fue superior a la reportada por Retamal et al. (2013); en T0 (fruto en condiciones normales) presentó una mayor firmeza de la pulpa, con 8,4kg, en comparación al T1 (fruto con molde), que presentó una firmeza de $6,6 \mathrm{~kg}$. El porcentaje de sólidos solubles totales para estos autores fue $\left(11,83-8,39^{\circ} \mathrm{Brix}\right)$. Resultados similares fueron encontrados por Monge (2016), en su investigación, con 70 genotipos de melón, principalmente, en peso promedio del fruto, producción por área, firmeza del fruto y porcentaje de sólidos solubles totales.

Los coeficientes de correlación entre las características evaluadas en 20 familias de medios hermanos de melón criollo ecuatoriano, se presentan en la tabla 3 .

Los resultados mostraron asociaciones fenotípicas altamente significativas positivas entre el carácter peso de fruto (PF) con diámetro de fruto (DF), longitud de fruto (LF), espesor de pulpa (EP), cavidad de fruto (CF) y producción por hectárea, indicando que la selección por mayor peso de fruto conduciría a un incremento del grosor de la pulpa, cavidad del fruto y producción por hectárea. No se observa una relación directa entre el peso del fruto (PF) con los caracteres grados brix (SS) y acidez de la pulpa (AP); con firmeza de la pulpa (FP), el peso del fruto presenta una correlación negativa no significativa.

El carácter (SS) sólidos solubles $\left({ }^{\circ} \mathrm{Brix}\right)$ presentó únicamente correlación positiva significativa con el carácter firmeza de la pulpa (FP). Los caracteres que presentaron asociaciones fenotípicas positivas significativas fueron grosor de la corteza (GC), con peso (PF), diámetro (DF) y longitud del fruto (LF). Esto demostró que existe una relación directa entre estas variables, por lo tanto, es posible mejorar el peso del fruto, si se direcciona la selección con base en cualquiera de los componentes del fruto de melón

Tabla 3. Coeficientes de correlación (r) entre las características evaluadas en 20 familias de medios hermanos de melón criollo ecuatoriano Cucumis melo var. dudaim (L.) Naudin.

\begin{tabular}{|c|c|c|c|c|c|c|c|c|c|}
\hline Carácter & $\begin{array}{l}\text { (DF) } \\
\mathrm{mm}\end{array}$ & $\begin{array}{l}\text { (LF) } \\
\mathrm{mm}\end{array}$ & $\begin{array}{l}\text { (GC) } \\
\mathrm{mm}\end{array}$ & $\begin{array}{l}(\mathrm{EP}) \\
\mathrm{mm}\end{array}$ & $\begin{array}{l}(\mathrm{CF}) \\
\mathrm{mm}\end{array}$ & $\begin{array}{l}\text { (SS) } \\
\left({ }^{\circ} \text { Brix }\right)\end{array}$ & $\begin{array}{l}\text { (AP) } \\
\mathrm{pH}\end{array}$ & $\begin{array}{l}(\mathrm{FP}) \\
\mathrm{kg} / \mathrm{cm}^{2}\end{array}$ & $\begin{array}{c}(\mathrm{P}) \\
\left(\mathrm{kg} \mathrm{ha}^{-1}\right)\end{array}$ \\
\hline$(\mathrm{PF}) \mathrm{g}$ & $0,69 * *$ & $0,67 * *$ & $0,25 *$ & $0,60 * *$ & $0,67 * *$ & $-0,30 *$ & $-0,28 *$ & $-0,06$ ns & $0,81 * *$ \\
\hline$(\mathrm{DF}) \mathrm{mm}$ & & $0,95^{* *}$ & $0,26^{*}$ & $0,90^{* *}$ & $0,83^{* *}$ & $-0,31^{*}$ & $-0,37 * *$ & $-0,20 \mathrm{~ns}$ & $0,54^{* *}$ \\
\hline$(\mathrm{LF}) \mathrm{mm}$ & & & $0,31 *$ & $0,87 * *$ & $0,78^{* *}$ & $-0,33 * *$ & $-0,39 * *$ & $-0,19 \mathrm{~ns}$ & $0,54 * *$ \\
\hline$(\mathrm{GC}) \mathrm{mm}$ & & & & $0,26^{*}$ & $0,14^{\mathrm{ns}}$ & $0,02^{\text {ns }}$ & $-0,34 * *$ & $-0,026$ & $0,07 \mathrm{~ns}$ \\
\hline$(\mathrm{EP}) \mathrm{mm}$ & & & & & $0,62^{* *}$ & $-0,35 * *$ & $-0,27^{*}$ & $-0,17 \mathrm{~ns}$ & $0,46^{* *}$ \\
\hline$(\mathrm{CF}) \mathrm{mm}$ & & & & & & $-0,31^{*}$ & $-0,33^{* *}$ & $-0,18 n s$ & $0,55^{* *}$ \\
\hline (SS) ( ${ }^{\circ}$ Brix) & & & & & & & $0,09^{\mathrm{ns}}$ & $0,40 * *$ & $-0,25^{*}$ \\
\hline$(\mathrm{AP}) \mathrm{pH}$ & & & & & & & & $0,25^{*}$ & $-0,19^{\mathrm{ns}}$ \\
\hline (FP) $\mathrm{kg} \mathrm{cm}^{2}$ & & & & & & & & & $-0,09^{\text {ns }}$ \\
\hline
\end{tabular}

$* *=$ significancia nivel $\mathrm{P} \leq 0,01, *=$ significancia nivel $\mathrm{P} \leq 0,05$ de probabilidad, $\mathrm{ns}=$ no significativo, $\mathrm{r}=$ coeficiente de correlación, peso del fruto (PF), diámetro del fruto (DF), longitud del fruto (LF), espesor de la pulpa (EP), grosor de la corteza (GC), cavidad del fruto (CF), firmeza de la pulpa (FP), acidez de la pulpa (AP), (SS) sólidos solubles $\left({ }^{\circ}\right.$ Brix) y $(\mathrm{P})$ producción por hectárea $\left(\mathrm{kg}\right.$ ha $\left.{ }^{-1}\right)$. 
citados. Espesor de la pulpa (EP) con grosor de la corteza (GC) y firmeza de la pulpa (FP) con acidez de la pulpa (AP); la existencia de correlación se puede atribuir a la presencia efectos pleiotrópicos de los genes, relación fisiológica y desarrollo, efecto ambiental o una combinación de todos los anteriores (Reddy et al. 2013). Los valores del coeficiente de correlación, se utilizan para encontrar asociaciones de importancia para un programa de mejoramiento.

Los promedios, coeficientes de variación genética, de variación ambiental, heredabilidad en sentido amplio y avance genético por selección de los caracteres evaluados en las 20 familias de medios hermanos de C. melo var. dudaim, se presentan en la tabla 4.
Los valores de los coeficientes de variación genotípica (CVg) para los caracteres evaluados en las 20 familias de medios hermanos, son mayores a los valores de los coeficientes de variación ambiental (CVa). Los parámetros de heredabilidad $\left(\mathrm{H}^{2}\right)$ son altos y el avance genético $(\Delta \mathrm{g})$ es de magnitud baja.

Para el carácter peso de fruto (PF), el (CVg) de 52,94\% y el (CVa) de $29,39 \%$, indican que, tanto el componente genético como el ambiental, poseen variaciones semejantes en la manifestación fenotípica de dicho carácter. Estos resultados son similares a los reportados por Kumar et al. (2013), quienes manifestaron que el peso promedio de la fruta registró altos valores de $\mathrm{CVa}$ y $\mathrm{CVg}$, lo

Tabla 4. Promedios, coeficiente de variación genética, coeficiente de variación ambiental, heredabilidad en sentido amplio y progreso genético esperado de las características evaluadas en 20 familias de medios hermanos de melón criollo ecuatoriano Cucumis melo var. dudaim (L.) Naudin.

\begin{tabular}{|c|c|c|c|c|c|}
\hline Caracteres & $\mathrm{CVg} \%$ & $\mathrm{CVa} \%$ & $\mathrm{H}^{2} \%$ & $\Delta \mathrm{g}$ & $\Delta \mathrm{g} \%$ \\
\hline$(\mathrm{PF}) \mathrm{g}$ & 52,94 & 29,39 & 90,68 & 71,10 & 23,01 \\
\hline$(\mathrm{DF}) \mathrm{mm}$ & 12,72 & 7,45 & 89,74 & 5,49 & 7,23 \\
\hline$(\mathrm{LF}) \mathrm{mm}$ & 18,68 & 11,70 & 88,44 & 7,92 & 9,64 \\
\hline$(\mathrm{EP}) \mathrm{mm}$ & 12,80 & 11,57 & 78,58 & $-0,03$ & $-0,18$ \\
\hline (GC) $\mathrm{mm}$ & 14,60 & 14,16 & 76,12 & 0,30 & 47,31 \\
\hline$(\mathrm{CF}) \mathrm{mm}$ & 23,04 & 8,46 & 95,70 & 6,48 & 15,84 \\
\hline (SS) ( ${ }^{\circ}$ Brix) & 14,48 & 8,87 & 88,89 & 1,65 & 16,21 \\
\hline$(\mathrm{AP}) \mathrm{pH}$ & 2,03 & 1,57 & 83,33 & $-0,06$ & $-1,01$ \\
\hline$(\mathrm{FP}) \mathrm{kg} \mathrm{cm}^{2}$ & 35,62 & 29,91 & 80,97 & 0,22 & 3,34 \\
\hline (P) $\left(\mathrm{kg} \mathrm{ha}^{-1}\right)$ & 55,49 & 46,32 & 81,15 & 608,78 & 34,67 \\
\hline
\end{tabular}

Coeficiente de variación genético $\mathrm{CVg} \%$, coeficiente de variación ambiental $\mathrm{CVa} \%$, heredabilidad en sentido amplio $\mathrm{h}_{\mathrm{A}}^{2} \%$, progreso genético esperado $\Delta \mathrm{g} \%$, diámetro del fruto (DF), longitud del fruto (LF), grosor de la corteza (GC), espesor de la pulpa (EP), cavidad del fruto (CF), (SS) sólidos solubles ( ${ }^{\circ}$ Brix), acidez de la pulpa (AP), firmeza de la pulpa (FP), (P) producción por hectárea $\left(\mathrm{kg}\right.$ ha $\left.{ }^{-1}\right)$.

que indica la presencia de un alto grado de variabilidad y un mejor alcance para la mejora de estos caracteres, mediante la selección. El carácter sólidos solubles (SS) presentó un (CVg) de 14,88\% y un (CVa) de 8,87\%, indicando moderada variación genética y baja variación ambiental en la expresión de este carácter, resultados similares a los reportados por Kumar et al. (2013), indicando que los sólidos solubles totales mostraron valores bajos a moderados de $\mathrm{CVa}$ y CVg, que indican la presencia de un alto grado de variabilidad $y$, por lo tanto, un mayor margen de selección. El carácter producción por hectárea $\left(\mathrm{kg} \mathrm{ha}^{-1}\right)$ presentó un alto $(\mathrm{CVg})$ de 52,49\% y un alto (CVa) de $46,32 \%$, indicando variaciones similares en la manifestación de este carácter, que favorecen el mejoramiento por selección.
Las estimaciones de heredabilidad en sentido amplio fueron de magnitud alta $(>60 \%)$ para todos los caracteres evaluados, indicando que la varianza genética juega un papel importante en la manifestación de la varianza fenotípica de los caracteres evaluados. Estas estimaciones coinciden con las encontradas por Kumar et al. (2013), quienes reportaron altos valores de heredabilidad para los caracteres de longitud del fruto $(83,92 \%)$, peso promedio del fruto $(90,35 \%)$, longitud de la cavidad del fruto $(89,41 \%)$, ancho de la cavidad del fruto $(66,93 \%)$, grosor de la corteza $(70,39 \%)$ y sólidos solubles totales $(86,51 \%)$. Rakhi \& Rajamony (2005) reportaron heredabilidad alta para longitud del fruto, circunferencia del fruto, peso promedio del fruto y producción por planta, lo que significó 
que la variabilidad de esos caracteres en alto porcentaje, se debe a la variación genética y podría haber mayor correspondencia entre los fenotipos y el genotipo de esos individuos. Reddy et al. (2013) encontraron alta heredabilidad para el carácter producción en melón, lo que implica que dicho carácter puede ser mejorado, a través de la selección.

Los valores estimados de progreso genético fueron de magnitud alta $(>60 \%)$ para los caracteres peso de la fruta $(\mathrm{PF})$, grosor de la corteza (GC) y rendimiento por hectárea; moderado, para los caracteres cavidad del fruto (CF) y (SS) sólidos solubles y baja $(<10 \%)$, en los demás caracteres evaluados, lo anterior, debido a heredabilidad sentido amplio presentada. Reddy et al. (2013) reportaron progresos genéticos diferentes para todos los caracteres, con excepción de peso y longitud del fruto. Indicaron que las estimaciones de avance genético fueron de gran magnitud $(>20 \%)$ para peso promedio del fruto $(57,27 \%)$, grosor de la corteza $(49,04 \%)$, sólidos solubles totales $(24,68 \%)$ y producción de fruto $(30,50 \%)$ y de magnitud moderada (10-20\%), para la longitud del fruto $(17,21 \%)$, diámetro del fruto $(10,02 \%)$ y espesor de pulpa $(13,51 \%)$.

La heredabilidad y avance genético fueron de magnitud alta para peso de fruto (PF) y producción por hectárea $\left(\mathrm{kg} \mathrm{ha}^{-1}\right)$; en cuanto a sólidos solubles (SS), aunque la heredabilidad es alta, el avance genético fue moderado, resultados que concuerdan con lo reportado Rakhi \& Rajamony (2005). El avance genético alcanzado es bueno, teniendo en cuenta la importancia agronómica y económica de estos caracteres. En general, la evaluación y la selección de familias de medios hermanos, como método de mejoramiento genético aplicado al cultivo de melón, permite identificar familias sobresalientes en cuanto a rendimiento y calidad de fruto, que podrían ser utilizadas para continuar con el proceso de mejoramiento genético en la especie.

Se concluye, que las familias seleccionadas de medios hermanos de melón criollo ecuatoriano fueron superiores al testigo (población original), en los 10 caracteres del fruto. Las 20 familias de medios hermanos presentaron alta variabilidad genética y alta heredabilidad en sentido amplio, para los caracteres relacionados con la calidad de fruto. Existe la posibilidad de alcanzar ganancias importantes mediante selección, especialmente, en los caracteres peso del fruto, sólidos solubles totales y rendimiento por hectárea, que son las características más importantes para el mercado.

Conflicto de intereses: El manuscrito fue preparado y revisado con la participación de todos los autores, quienes declaramos que no existe ningún conflicto de intereses que ponga en riesgo la validez de los resultados presentados.

\section{REFERENCIAS}

1. ABRAHAM-JUÁREZ, M. DEL R.; ESPITIA-VÁZQUEZ, I.; GUZMÁN-MENDOZA, R.; OLALDE-PORTUGAL, V.; RUIZ-AGUILAR, G.M.L; GARCÍA-HERNÁNDEZ, J.L.; HERRERA-ISIDRÓN, L.; NÚÑEZ-PALENIUS, H.G. 2018. Development, yield, and quality of melon fruit
(Cucumis melo L.) inoculated with mexican native strains of Bacillus subtilis (Ehrenberg). Agrociencia. 52(1):91-102.

2. ARAGÃO, F.; NUNES, G.; QUEIRÓZ, M. 2015. Genotype x environment interaction of melon families based on fruit quality traits. Crop Breeding and Applied Biotechnology. 15(2):79-86. https://doi.org/10.1590/1984-70332015v15n2a15

3. ARAMENDIZ, H.; CARDONA, C.; VERGARA, C. 2014. Genetic parameters related to fruit characteristics in eggplant (Solanum melongena L.). Rev. Col. Ciencias Hortícolas. 8(1):103-111.

4. CANO-RÍOS, P.; THERAN-KRUGE K.; ESPARZAMARTÍNEZ, J. 2004. Calidad de fruta de híbridos de melón reticulado (Cucumis melo L.) bajo condiciones de la Comarca Lagunera. Revista Chapingo Serie Zonas Áridas. 3:123-130.

5. DÍAZ-ALVARADO, J.M.; MONGE-PÉREZ, J.E. 2017. Efecto de la poda y la densidad de siembra sobre el rendimiento y calidad de melón Cantaloupe (Cucumis melo L.) cultivado bajo invernadero. Rev. Col. Ciencias Hortícolas, 11(1):2129.

https://doi.org/10.17584/rcch.2017v11i1.5742

6. EGUIARTE, L.; AGUIRRE, J.; JARDÓN, L.; AGUIRRE, E.; SOUZA, V. 2015. Genómica de Poblaciones: nada en evolución va a tener sentido si no es a la luz de la genómica, y nada en genómica tendrá sentido si no es a la luz de la evolución. TIP. 16(1):42-56. https://doi.org/10.1016/S1405-888X(13)72077-1

7. FAO. 2018. FAOSTAT. Roma. Disponible desde Internet en: http://www.fao.org/faostat/en/\#data/QC (con acceso el 22/09/2019).

8. GOBIERNO AUTÓNOMO DESCENTRALIZADO DEL CANTÓN QUEVEDO. 2014. Plan de desarrollo y de ordenamiento territorial. (D. de P. y G. Municipal, Ed.). Quevedo: Gobierno autónomo descentralizado del cantón Quevedo.

9. GUIS, M.; ROUSTAN, J.; DOGIMONT, C.; PITRAT, M.; PECH, J. 1998. Melon biotechnology. Biotechnology and Genetic Engineering Reviews. 15:289-311.

10. INSTITUTO NACIONAL DE ESTADÍSTICA Y CENSOS, INEC. 2017. III Censo Nacional Agropecuario. Resumen 2017, Disponible desde Internet en: https://www.ecuadorencifras.gob.ec/censo-nacionalagropecuario (con acceso 08/11/2019).

11. INSTITUTO NACIONAL DE METEOROLOGIA E HIDROLOGIA, INAMHI. 2018. Boletín climático 
semestral 2018. Quito: INAMHI. Disponible desde Internet en:

http:/ / www.serviciometeorologico.gob.ec/clima (con acceso 08/11/2019).

12. KUMAR, B.P.; BEGUM, H.; REDDY, M.T.; BABU, J.D.; SUBBARAMA, R.; REDDY, K. 2013. Correlation and path coefficient analysis in muskmelon (Cucumis melo L.). Suranaree J. Sci. Technol. 20(2):135-149. https://doi.org/10.20546/ijcmas.2017.606.268

13. MONGE, J. 2016. Evaluación de 70 genotipos de melón (Cucumis melo L.) cultivados bajo invernadero en Costa Rica. InterSedes. 17(36):73-112.

https://doi.org/10.15517/isucr.v17i36.26944

14. PARIS, H.; AMAR, Z.; LEV, E. 2012. Medieval History of the Duda'im Melon (Cucumis melo, Cucurbitaceae). Economic Botany. 66(3):276-284. https://doi.org/10.1007/s12231-012-9205-4

15. PEÑA, A.; GUERRERO, H.; RODRIGUEZ, J.; SAHAGUN, J.; MAGAÑA, N. 2013. Selección temprana en familias de medios hermanos maternos de tomate de cascara de la raza Puebla. Revista Chapingo, Serie Horticultura. 19(1):5-13. http://dx.doi.org/10.5154/r.rchsh.2012.01.18

16. PÉREZ ZAMORA, O.; CIGALES RIVERO, M.R.; PÉREZ CASTRO, K.G. 2007. Nitrógeno y humedad del suelo, concentración nutrimental, rendimiento y calidad de melón cantaloupe. Terra Latinoamericana. 25(2):177-185.

17. PISTORALE, S.M.; ABBOTT, L.A.; ANDRÉS, A. 2008. Diversidad genética y heredabilidad en sentido amplio en agropiro alargado, Thinopyrum ponticum. Cien. Inv. Agr., 35(3):259-264. https://doi.org/10.4067/S0718-16202008000300003

18. PITRAT, M.; HANELT, P.; HAMMER, K. 2000. Some comments on infraspecific classification of cultivars of melon. Acta Hort. 510:29-36.

19. RAKHI, R.; RAJAMONY, L. 2005. Short communication Variability, etalheritability and genetic advance in landraces of culinary melon (Cucumis melo L.). J. Tropical Agriculture. 43(1-2):79-82.
20. REDDY, B.; BEGUM, H.; SUNIL, N.; REDDY, M. 2013. Variance component analysis of quantitative raits in muskmelon (Cucumis melo L.). Trakia J. of Sciences. 11(2):118-124.

21. RETAMAL, A.; VÁSQUEZ, L.; MAZUELA, P. 2013. Efecto de la transformación geométrica del fruto de melón (Cucumis melo L) sobre su calidad bajo cultivo sin suelo. Idesia (Arica). 30(3):65-69. https://doi.org/10.4067/s0718-34292012000300008

22. SEBASTIAN, P.; SCHAEFER, H.; TELFORD, I.; RENNER, S. 2010. Cucumber (Cucumis sativus) and melon (C. melo) have numerous wild relatives in Asia and Australia, and the sister species of melon is from Australia. Proc. Nal Acad. Scienc. 107(32):14269-14273. https://doi.org/10.1073/pnas.1005338107

23. SOLTANI, F.; KASHI, A.; AKASHI, Y.; MOSTOFI, Y.; KATO, K.; ZAMANI, Z. 2010. Characterization of Iranian melon landraces of Cucumis melo L. Groups Flexuosus and Dudaim by analysis of morphological characters and random amplified polymorphic DNA. Breeding Science, 60(1):34-45. https://doi.org/10.1270/jsbbs.60.34

24. VALLEJO, F.; ESTRADA, E. 2004. El cultivo del melón Cucumis melo L. In Producción de hortalizas de clima cálido (p.239310). Palmira. Valle del Cauca: Universidad Nacional de Colombia - Sede Palmira. Disponible desde Internet en: http://bdigital.unal.edu.co/46253/18/958809528_Part05. PDF (con acceso 07/04/2019).

25. WANG, Y.; WU, D.; HUANG, J.; TSAO, S.; HWU, K.; LO, H. 2016. Mapping quantitative trait loci for fruit traits and powdery mildew resistance in melon (Cucumis melo). Botanical Studies. 57(1):19. https://doi.org/10.1186/s40529-016-0130-1 